\title{
Weakly distance-regular digraphs of valency three, I
}

\author{
Yuefeng Yang \\ Sch. Math. Sci. \& Lab. Math. Com. Sys., \\ Beijing Normal University, \\ Beijing, 100875, China \\ yangyf@mail . bnu . edu.cn
}

\author{
Benjian Lv \\ Sch. Math. Sci. \& Lab. Math. Com. Sys., \\ Beijing Normal University, \\ Beijing, 100875, China \\ bjlv@bnu.edu.cn
}

\author{
Kaishun Wang \\ Sch. Math. Sci. \& Lab. Math. Com. Sys., \\ Beijing Normal University, \\ Beijing, 100875, China \\ wangks@bnu . edu.cn
}

Submitted: Jul 15, 2015; Accepted: Mar 30, 2016; Published: Apr 15, 2016

Mathematics Subject Classifications: 05E30

\begin{abstract}
Suzuki (2004) classified thin weakly distance-regular digraphs and proposed the project to classify weakly distance-regular digraphs of valency 3 . The case of girth 2 was classified by the third author (2004) under the assumption of the commutativity. In this paper, we continue this project and classify these digraphs with girth more than 2 and two types of arcs.
\end{abstract}

Keywords: Weakly distance-regular digraph; Cayley digraph

\section{Introduction}

A digraph $\Gamma$ is a pair $(X, A)$ where $X$ is a finite set of vertices and $A \subseteq X^{2}$ is a set of arcs. Throughout this paper we use the term 'digraph' to mean a finite directed graph with no loops. We write $V \Gamma$ for $X$ and $A \Gamma$ for $A$. A path of length $r$ from $u$ to $v$ is a finite sequence of vertices $\left(u=w_{0}, w_{1}, \ldots, w_{r}=v\right)$ such that $\left(w_{t-1}, w_{t}\right) \in A \Gamma$ for $t=1,2, \ldots, r$. A digraph is said to be strongly connected if, for any two distinct vertices $x$ and $y$, there is a path from $x$ to $y$. The length of a shortest path from $x$ to $y$ is called the distance from $x$ to $y$ in $\Gamma$, denoted by $\partial_{\Gamma}(x, y)$. The diameter of $\Gamma$ is the maximum value of the distance function in $\Gamma$. Let $\widetilde{\partial}_{\Gamma}(x, y)=\left(\partial_{\Gamma}(x, y), \partial_{\Gamma}(y, x)\right)$ and $\widetilde{\partial}(\Gamma)=\left\{\widetilde{\partial}_{\Gamma}(x, y) \mid x, y \in V \Gamma\right\}$. If no confusion occurs, we write $\partial(x, y)(\operatorname{resp} . \widetilde{\partial}(x, y))$ instead of $\partial_{\Gamma}(x, y)\left(\operatorname{resp} . \widetilde{\partial}_{\Gamma}(x, y)\right)$. An $\operatorname{arc}(u, v)$ of $\Gamma$ is of type $(1, r)$ if $\partial(v, u)=r$. A path $\left(w_{0}, w_{1}, \ldots, w_{r-1}\right)$ is said to be a 
circuit of length $r$ if $\partial\left(w_{r-1}, w_{0}\right)=1$. A circuit is undirected if each of its arcs is of type $(1,1)$. The girth of $\Gamma$ is the length of a shortest circuit.

Let $\Gamma=(X, A)$ and $\Gamma^{\prime}=\left(X^{\prime}, A^{\prime}\right)$ be two digraphs. $\Gamma$ and $\Gamma^{\prime}$ are isomorphic if there is a bijection $\sigma$ from $X$ to $X^{\prime}$ such that $(x, y) \in A$ if and only if $(\sigma(x), \sigma(y)) \in A^{\prime}$. In this case, $\sigma$ is called an isomorphism from $\Gamma$ to $\Gamma^{\prime}$. An isomorphism from $\Gamma$ to itself is called an automorphism of $\Gamma$. The set of all automorphisms of $\Gamma$ forms a group which is called the automorphism group of $\Gamma$ and denoted by $\operatorname{Aut}(\Gamma)$. A digraph $\Gamma$ is vertex transitive if Aut $(\Gamma)$ is transitive on $V \Gamma$.

Lam [5] introduced a concept of distance-transitive digraphs. A strongly connected digraph $\Gamma$ is said to be distance-transitive if, for any vertices $x, y, x^{\prime}$ and $y^{\prime}$ of $\Gamma$ satisfying $\partial(x, y)=\partial\left(x^{\prime}, y^{\prime}\right)$, there exists an automorphism $\sigma$ of $\Gamma$ such that $x^{\prime}=\sigma(x)$ and $y^{\prime}=\sigma(y)$. Damerell [4] generalized this concept to that of distance-regular digraphs. He showed that the girth $g$ of a distance-regular digraph of diameter $d$ is either $2, d$ or $d+1$, and the one with $d=g$ is a coclique extension of a distance-regular digraph with $d=g-1$. Bannai, Cameron and Kahn [2] proved that a distance-transitive digraph of odd girth is a Paley tournament or a directed cycle. Leonard and Nomura [6] proved that except directed cycles all distance-regular digraphs with $d=g-1$ have girth $g \leqslant 8$. In order to find 'better' classes of digraphs with unbounded diameter, Damerell [4] also proposed a more natural definition of distance-transitivity, i.e., weakly distance-transitivity. In [8], Wang and Suzuki introduced weakly distance-regular digraphs as a generalization of distance-regular digraphs and weakly distance-transitive digraphs.

A strongly connected digraph $\Gamma$ is said to be weakly distance-transitive if, for any vertices $x, y, x^{\prime}$ and $y^{\prime}$ satisfying $\widetilde{\partial}(x, y)=\widetilde{\partial}\left(x^{\prime}, y^{\prime}\right)$, there exists an automorphism $\sigma$ of $\Gamma$ such that $x^{\prime}=\sigma(x)$ and $y^{\prime}=\sigma(y)$. A strongly connected digraph $\Gamma$ is said to be weakly distance-regular if, for all $\widetilde{h}, \widetilde{i}, \widetilde{j} \in \widetilde{\partial}(\Gamma)$ and $\widetilde{\partial}(x, y)=\widetilde{h}$, the number $p_{\tilde{i}, \tilde{j}}^{\widetilde{h}}:=\left|P_{\tilde{i}, \tilde{j}}(x, y)\right|$ depends only on $\widetilde{h}, \widetilde{i}, \widetilde{j}$, where

$$
P_{\widetilde{i}, \tilde{j}}(x, y)=\{z \in V \Gamma \mid \widetilde{\partial}(x, z)=\widetilde{i} \text { and } \widetilde{\partial}(z, y)=\widetilde{j}\} \text {. }
$$

The nonnegative integers $p_{i, \tilde{j}}^{\widetilde{h}}$ are called the intersection numbers. We say that $\Gamma$ is commutative (resp. thin) if $p_{\tilde{i}, \tilde{j}}^{\widetilde{h}}=p_{\tilde{j}, \tilde{i}}^{\widetilde{h}}\left(\operatorname{resp} . p_{i, \widetilde{j}}^{\widetilde{h}} \leqslant 1\right)$ for all $\widetilde{i}, \widetilde{j}, \widetilde{h} \in \widetilde{\partial}(\Gamma)$. Note that a weakly distance-transitive digraph is weakly distance-regular.

Let $G$ be a finite group and $S$ a subset of $G$ not containing the identity. The Cayley digraph $\Gamma=\operatorname{Cay}(G, S)$ is a digraph with the vertex set $G$ and the arc set $\{(x, s x) \mid x \in$ $G, s \in S\}$.

In [8], Wang and Suzuki determined all commutative 2-valent weakly distance-regular digraphs. In [7], Suzuki determined all thin weakly distance-regular digraphs and proved the nonexistence of noncommutative weakly distance-regular digraphs of valency 2 . Moreover, he proposed the project to classify weakly distance-regular digraphs of valency 3 . In [9], Wang classified all commutative weakly distance-regular digraphs of valency 3 and girth 2. In this paper, we continue this project, and obtain the following result.

Theorem 1. Let $\Gamma$ be a weakly distance-regular digraph of valency 3 and girth more than 2. If $\Gamma$ has two types of arcs, then $\Gamma$ is isomorphic to one of the following digraphs: 
(i) $\operatorname{Cay}\left(\mathbb{Z}_{4} \times \mathbb{Z}_{g},\{(0,1),(2,1),(1,0)\}\right)$, where $g=3$ or $g \geqslant 5$.

(ii) $\Gamma_{q, 2 m q, 1}, \Gamma_{q, m q+2, q}$ or $\Gamma_{q, 2 m q-2 q+2 t, q+1-t}$ in Construction 3, where $q \geqslant 3, m \geqslant 1$ and $2 \leqslant t \leqslant q-1$.

This paper is organized as follows. In Section 2, we construct two families of weakly distance-regular digraphs of valency 3. In Section 3, we discuss some properties for circuits of weakly distance-regular digraphs. In Section 4, we prove our main theorem.

\section{Constructions}

In this section, we construct two families of weakly distance-regular digraphs of valency 3 . For any element $x$ in a residue class ring, we assume that $\hat{x}$ denotes the minimum nonnegative integer in $x$. Denote $\beta(w)=\left(1+(-1)^{w+1}\right) / 2$ for any integer $w$.

Proposition 2. Let $g \geqslant 3$. Then $\Gamma_{g}:=\operatorname{Cay}\left(\mathbb{Z}_{4} \times \mathbb{Z}_{g},\{(1,0),(0,1),(2,1)\}\right)$ is a weakly distance-regular digraph if and only if $g \neq 4$.

Proof. For any vertex $(a, b)$ distinct with $(0,0)$, we have

$$
\widetilde{\partial}((0,0),(a, b))= \begin{cases}(\hat{a}, 4-\hat{a}), & \text { if } b=0 \\ (\hat{b}+\beta(\hat{a}), g-\hat{b}+\beta(\hat{a})), & \text { if } b \neq 0 .\end{cases}
$$

Suppose $g \neq 4$. We will show that $\Gamma_{g}$ is weakly distance-transitive. Let $(a, b)$ and $(x, y)$ be any two vertices satisfying $\widetilde{\partial}((0,0),(a, b))=\widetilde{\partial}((0,0),(x, y))$. It suffices to verify that there exists an automorphism $\sigma$ of $\Gamma_{g}$ such that $\sigma(0,0)=(0,0)$ and $\sigma(a, b)=(x, y)$. If $(a, b)=(x, y)$, then the identity permutation is a desired automorphism. Now suppose $(a, b) \neq(x, y)$. Then $b \neq 0, y \neq 0$ and $(\hat{b}+\beta(\hat{a}), g-\hat{b}+\beta(\hat{a}))=(\hat{y}+\beta(\hat{x}), g-\hat{y}+\beta(\hat{x}))$. It follows that $b=y$ and $a-x=2$. Let $\sigma$ be the permutation on $V \Gamma_{g}$ such that

$$
\sigma(u, v)= \begin{cases}(u, v), & \text { if } v \neq b \\ (u+2, v), & \text { if } v=b\end{cases}
$$

Routinely, $\sigma$ is a desired automorphism.

In $\Gamma_{4}, \widetilde{\partial}((0,0),(0,2))=\widetilde{\partial}((0,0),(2,0))=(2,2)$. But $P_{(1,3),(3,3)}((0,0),(0,2))=\{(1,0)\}$ and $P_{(1,3),(3,3)}((0,0),(2,0))=\emptyset$. Hence, $\Gamma_{4}$ is not a weakly distance-regular digraph.

Construction 3. Let $q, s, k$ be integers with $q>2, s>2$ and $\max \{1, q-s+2\} \leqslant k \leqslant q$. Write $s=2 m q+p$ with $m \geqslant 0$ and $0 \leqslant p<2 q$. Let $\Gamma_{q, s, k}$ be the digraph with the vertex set $\mathbb{Z}_{q} \times \mathbb{Z}_{s}$ whose arc set consists of $((a, b),(a+1, b)),((a, c),(a, c+1)),((a, d),(a+1, d-1))$, $((a,-1),(a-k+1,0))$ and $((a, 0),(a+k,-1))$, where $a \in \mathbb{Z}_{q}, b, c, d \in \mathbb{Z}_{s}, \hat{c} \neq s-1$ and $d \neq 0$. See Figure 1.

In the following, we will prove that $\Gamma_{q, s, k}$ is a weakly distance-regular digraph if and only if one of the following holds:

$\mathrm{C} 1: p=0$ and $k=1$.

C2: $p=q+2$ or $p=2$, and $k=q$.

C3: $4 \leqslant p \leqslant 2 q-2, p$ is even and $k=q+1-p / 2$. 


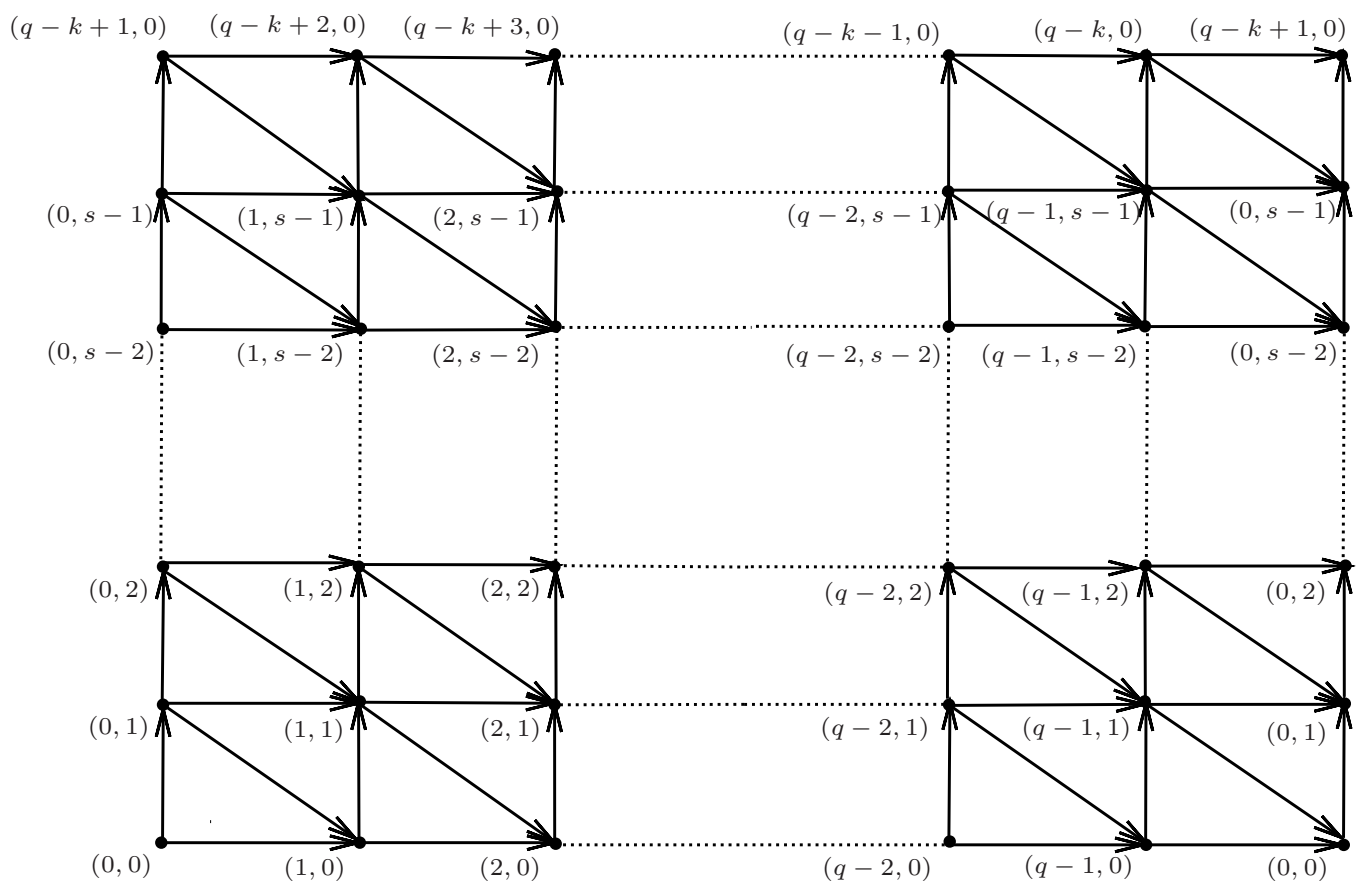

Figure 1: The digraph $\Gamma_{q, s, k}$.

Lemma 4. $\Gamma_{q, s, k}$ is a vertex transitive digraph.

Proof. Pick any vertex $(a, b)$. It suffices to show that there exists an automorphism $\sigma$ of $\Gamma_{q, s, k}$ such that $\sigma(0,0)=(a, b)$. Let $\sigma$ be the permutation on $V \Gamma_{q, s, k}$ such that

$$
\sigma(x, y)= \begin{cases}(x+a, y+b), & \text { if } \hat{y} \in\{0,1,2, \ldots, s-1-\hat{b}\} \\ (x+a-k+1, y+b), & \text { otherwise. }\end{cases}
$$

Routinely, $\sigma$ is a desired automorphism.

For any two integers $i$ and $j$, we write $i \equiv j$ instead of $i \equiv j(\bmod q)$. For any vertex $(a, b)$ of $\Gamma_{q, s, k}$, let $f(a, b), g(a, b)$ and $h(a)$ be nonnegative integers less than $q$ such that

$$
f(a, b) \equiv \hat{a}+\hat{b}-k-p+1, g(a, b) \equiv q-\hat{a}-\hat{b} \text { and } h(a) \equiv k-\hat{a}-1 .
$$

By the structure of $\Gamma_{q, s, k}$, we have

$$
\widetilde{\partial}((0,0),(a, b))=(\min \{\hat{a}+\hat{b}, s-\hat{b}+f(a, b)\}, \min \{\hat{b}+g(a, b), s-\hat{b}+h(a)\}) .
$$

Lemma 5. Let $\mathrm{C} 1, \mathrm{C} 2$ or $\mathrm{C} 3$ hold. In $\Gamma_{q, s, k}, \partial((0,0),(a, b))=\hat{a}+\hat{b}$ if and only if $\partial((a, b),(0,0))=\hat{b}+g(a, b)$.

Proof. Let $M=s-2 \hat{b}-\hat{a}+f(a, b), N=s-2 \hat{b}+h(a)-g(a, b)$ and $\hat{b}=n^{\prime} q+r^{\prime}$ with $0 \leqslant r^{\prime}<q$. By (2), we only need to prove $M \geqslant 0$ if and only if $N \geqslant 0$. From (1), note that $f(a, b)+g(a, b)$ equals to $k-1$ or $q+k-1$, and $h(a)$ equals to $k-\hat{a}-1$ or $q+k-\hat{a}-1$. 
Case 1. $f(a, b)+g(a, b)=k-1$ and $h(a)=k-\hat{a}-1$, or $f(a, b)+g(a, b)=q+k-1$ and $h(a)=q+k-\hat{a}-1$.

In this case, it is routine to check $M=N$, as desired.

Case 2. $f(a, b)+g(a, b)=k-1$ and $h(a)=q+k-\hat{a}-1$.

In this case, only $\mathrm{C} 1$ or $\mathrm{C} 3$ holds by $k<\hat{a}+1$.

Assume that C1 holds. Then $f(a, b)=g(a, b)=0$ and $h(a)=q-\hat{a}$, which imply that $\hat{a}+r^{\prime}=0$ or $q$. Since $h(a)<q$, one gets $\hat{a} \neq 0$. Hence, $\hat{a}+r^{\prime}=q$. Then $M=2\left(m-n^{\prime}\right) q-q-r^{\prime} \geqslant 0$ if and only if $N=2\left(m-n^{\prime}\right) q-r^{\prime} \geqslant 0$.

Assume that C3 holds. Then $f(a, b)+g(a, b)=q-p / 2$ and $h(a)=2 q-p / 2-\hat{a}$. By (1), note that $g(a, b)=q-\hat{a}-r^{\prime}$ or $2 q-\hat{a}-r^{\prime}$, and $p / 2+\hat{a}>q$. Suppose $g(a, b)=q-\hat{a}-r^{\prime}$. Then $f(a, b)=\hat{a}+r^{\prime}-p / 2$. From $\hat{a}+r^{\prime} \leqslant q$, we have $0<p / 2-r^{\prime}<q$, which implies $M=2\left(m-n^{\prime}\right) q+p / 2-r^{\prime} \geqslant 0$ if and only if $N=2\left(m-n^{\prime}\right) q+q+p / 2-r^{\prime} \geqslant 0$. Suppose $g(a, b)=2 q-\hat{a}-r^{\prime}$. Since $f(a, b)=\hat{a}-q+r^{\prime}-p / 2$, we have $0 \leqslant r^{\prime}-p / 2<q$, which implies $M=2\left(m-n^{\prime}\right) q-q+p / 2-r^{\prime} \geqslant 0$ if and only if $N=2\left(m-n^{\prime}\right) q+p / 2-r^{\prime} \geqslant 0$.

Case 3. $f(a, b)+g(a, b)=q+k-1$ and $h(a)=k-\hat{a}-1$.

In this case, only $\mathrm{C} 1$ or $\mathrm{C} 3$ holds by $f(a, b)+g(a, b) \leqslant 2(q-1)$.

Assume that $\mathrm{C} 1$ holds. Then $h(a)=\hat{a}=0$ and $r^{\prime} \neq 0$, which imply that $f(a, b)=r^{\prime}$ and $g(a, b)=q-r^{\prime}$. Then $M=2\left(m-n^{\prime}\right) q-r^{\prime} \geqslant 0$ if and only if $N=2\left(m-n^{\prime}\right) q-q-r^{\prime} \geqslant 0$.

Assume that C3 holds. Then $f(a, b)+g(a, b)=2 q-p / 2$ and $h(a)=q-p / 2-\hat{a}$. By (1), note that $g(a, b)=q-\hat{a}-r^{\prime}$ or $2 q-\hat{a}-r^{\prime}$, and $p / 2+\hat{a} \leqslant q$. Suppose $g(a, b)=$ $q-\hat{a}-r^{\prime}$. Then $f(a, b)=q+\hat{a}+r^{\prime}-p / 2$ and $0 \leqslant q+r^{\prime}-p / 2<q$, which imply $M=2\left(m-n^{\prime}+1\right) q-q-r^{\prime}+p / 2 \geqslant 0$ if and only if $N=2\left(m-n^{\prime}\right) q+p / 2-r^{\prime} \geqslant 0$. Suppose $g(a, b)=2 q-\hat{a}-r^{\prime}$. Since $f(a, b)=\hat{a}+r^{\prime}-p / 2$, we have $p / 2-r^{\prime} \leqslant \hat{a}<q$, which implies $M=2\left(m-n^{\prime}\right) q+p / 2-r^{\prime} \geqslant 0$ if and only if $N=2\left(m-n^{\prime}\right) q-q+p / 2-r^{\prime} \geqslant 0$.

Thus, desired result follows.

Lemma 6. If $\mathrm{C} 1, \mathrm{C} 2$ or $\mathrm{C} 3$ holds, then $\Gamma_{q, s, k}$ is a weakly distance-regular digraph.

Proof. We will prove that $\Gamma_{q, s, k}$ is weakly distance-transitive. Let $(a, b)$ and $(x, y)$ be two vertices satisfying $\widetilde{\partial}((0,0),(a, b))=\widetilde{\partial}((0,0),(x, y))$. It suffices to find $\sigma \in \operatorname{Aut}\left(\Gamma_{q, s, k}\right)$ such that $\sigma(0,0)=(0,0)$ and $\sigma(a, b)=(x, y)$. By $(2)$, we divide the proof into two cases.

Case 1. $\partial((0,0),(a, b))=\hat{a}+\hat{b}$.

Suppose $\partial((0,0),(x, y))=\hat{x}+\hat{y}$. Then $g(a, b)=g(x, y)$. By Lemma 5 , we have $\hat{b}+g(a, b)=\hat{y}+g(x, y)$. This implies that $a=x$ and $b=y$. Hence, the identity permutation is a desired automorphism.

Suppose $\partial((0,0),(x, y))=s-\hat{y}+f(x, y)$. Then $\hat{a}+\hat{b}=s-\hat{y}+f(x, y)$, which implies $\hat{x} \equiv \hat{a}+\hat{b}+k-1$ by $s \equiv p$. Hence, $\hat{x}=f(a, b)$ and $g(a, b)=h(x)$. From Lemma 5 , we have $\hat{b}+g(a, b)=s-\hat{y}+h(x)$. This implies $\hat{y}=s-\hat{b}$. Let $\sigma$ be the permutation on $V \Gamma_{q, s, k}$ such that

$$
\sigma(u, v)= \begin{cases}(u, v), & \text { if } v=0 \\ (f(u, v),-v), & \text { if } v \neq 0\end{cases}
$$

Routinely, $\sigma$ is a desired automorphism. 
Case 2. $\partial((0,0),(a, b))=s-\hat{b}+f(a, b)$.

Suppose $\partial((0,0),(x, y))=s-\hat{y}+f(x, y)$. Then $\hat{y}-\hat{b}=f(x, y)-f(a, b)$. We have $\hat{y}-\hat{b} \equiv \hat{x}+\hat{y}-\hat{a}-\hat{b}$. This implies $x=a$. By Lemma 5 , one gets $s-\hat{b}+h(a)=s-\hat{y}+h(x)$, which implies that $y=b$. Hence, the identity permutation is a desired automorphism.

Suppose $\partial((0,0),(x, y))=\hat{x}+\hat{y}$. It is similar to Case 1 and the desired result holds.

By Lemma 4 , for vertices $(a, b)$ and $(x, y)$ of $\Gamma_{q, s, k}$, we have

$$
\widetilde{\partial}((a, b),(x, y))= \begin{cases}\widetilde{\partial}((0,0),(x-a, y-b)), & \text { if } \hat{y} \in\{\hat{b}, \hat{b}+1, \ldots, s-1\}, \\ \widetilde{\partial}((0,0),(x-a+k-1, y-b)), & \text { otherwise. }\end{cases}
$$

Lemma 7. If $\Gamma_{q, s, k}$ is weakly distance-regular, then $\mathrm{C} 1, \mathrm{C} 2$ or $\mathrm{C} 3$ holds.

Proof. Suppose for the contrary that C1, C2 and C3 do not hold. Let $e=(0,0), z=(0,1)$, $w=(k, s-1)$ be the vertices of $\Gamma_{q, s, k}$, and $\alpha(v)=\left(3+(-1)^{v}\right) / 4$ for $v \in \mathbb{Z}$. By $(2)$, note that $\widetilde{\partial}(e, z)=\widetilde{\partial}(e, w)=(1, q)$. To prove this lemma, we would pick proper $x, y \in V \Gamma_{q, s, k}$ such that $\widetilde{\partial}(e, x)=\widetilde{\partial}(e, y)$, and

$$
P_{(1, q), \widetilde{\partial}(z, x)}(e, y)=\emptyset \quad \text { or } \quad P_{(1, q), \widetilde{\partial}(w, x)}(e, y)=\emptyset,
$$

which contrary to $z \in P_{(1, q), \widetilde{\partial}(z, x)}(e, x)$ or $w \in P_{(1, q), \widetilde{\partial}(w, x)}(e, x)$.

Case 1. $k \neq q$ and $2 k+p \geqslant 2(q-\alpha(p)+2)$.

Let $x=(k, \alpha(s)-1+s / 2)$ and $y=(k, \alpha(s)+s / 2)$. In this case, $\widetilde{\partial}(e, x)=\widetilde{\partial}(e, y)=$ $\widetilde{\partial}(z, y)=((m+1) q+1,(m+2) q-k)$ and $\widetilde{\partial}(w, x)=((m+1) q,-\alpha(s)+s / 2)$. Since $\partial(y, w)=-\alpha(s)+s / 2-1$, we have (3) holds.

Case 2. $k \neq q, p \geqslant q$ and $2 k+p \leqslant 2(q-\alpha(p)+1)$.

Let $x=(0, \alpha(s)+s / 2)$ and $y=(q+\alpha(p)+1-k-p / 2,(m-1) q+p+k-1)$. In this case, $\widetilde{\partial}(e, x)=\widetilde{\partial}(e, y)=(\alpha(s)+s / 2, k-\alpha(s)-1+s / 2)$ and $\widetilde{\partial}(z, x)=(\alpha(s)-1+s / 2, k-$ $\alpha(s)+s / 2)$. Since $\widetilde{\partial}(z, y)=(\alpha(s)-1+s / 2, k-\alpha(s)-1+s / 2)$ and $\partial(w, y)=\alpha(s)+s / 2$, we have $(3)$ holds.

Case 3. $k \neq q, p<q$ and $2(\alpha(p)+1)<2 k+p \leqslant 2(q-\alpha(p)+1)$.

Let $x=(0, \alpha(s)+s / 2)$ and $y=(q+\alpha(p)+1-k-p / 2,(m-1) q+p+k-1)$. In this case, $\widetilde{\partial}(e, x)=\widetilde{\partial}(e, y)=(\alpha(s)+s / 2, k-\alpha(s)-1+s / 2)$ and $\partial(w, y)=\alpha(s)+s / 2$. If $p=2(1-\alpha(p))$, then $\widetilde{\partial}(z, x)=(\alpha(s)-1+s / 2, \alpha(s)-1+s / 2)$ and $\widetilde{\partial}(z, y)=(\alpha(s)-1+$ $s / 2,(m-1) q+p+k-2)$; if $p \neq 2(1-\alpha(p))$, then $\widetilde{\partial}(z, x)=(\alpha(s)-1+s / 2, k-\alpha(s)+s / 2)$ and $\widetilde{\partial}(z, y)=(\alpha(s)-1+s / 2, k-\alpha(s)-1+s / 2)$. Hence, (3) holds.

Case $4 . k \neq q, p<q$ and $2 k+p \leqslant 2(\alpha(p)+1)$.

Let $x=(k, \alpha(s)-1+s / 2)$ and $y=(k, \alpha(s)+s / 2)$. In this case, $\widetilde{\partial}(e, x)=\widetilde{\partial}(e, y)=$ $\widetilde{\partial}(z, y)=(m q+1, q-\alpha(s)-1+s / 2)$ and $\widetilde{\partial}(w, x)=(m q,-\alpha(s)+s / 2)$. Since $\partial(y, w)=$ $-\alpha(s)-1+s / 2$, we have (3) holds.

Case 5. $k=q$ and $p \geqslant q+3$.

Let $x=(q-1,(m-1) q+p)$ and $y=(0,(m+1) q)$. In this case, $\widetilde{\partial}(e, x)=\widetilde{\partial}(e, y)=$ $((m+1) q,(m+1) q)$ and $\partial(z, x)=m q+p-2$. Since $\partial(z, y)=(m+1) q-1$ and $\partial(w, y)=(m+1) q$, we have (3) holds. 
Case 6. $k=q$ and $3 \leqslant p \leqslant q+1$.

Let $x=(q-2, m q+2)$ and $y=(q-2, m q+1)$. In this case, $\widetilde{\partial}(e, x)=\widetilde{\partial}(e, y)=$ $\widetilde{\partial}(z, x)=((m+1) q-1, m q+2)$. Since $\widetilde{\partial}(z, y)=((m+1) q-2, m q+2)$ and $\partial(y, w)=m q+3$, we have (3) holds.

Case 7. $k=q$ and $p \leqslant 1$.

Let $x=(q-1, m q+p)$ and $y=(0, m q)$. In this case, $\widetilde{\partial}(e, x)=\widetilde{\partial}(e, y)=(m q, m q)$ and $\partial(z, x)=(m+1) q+p-2$. Since $\partial(z, y)=m q-1$ and $\partial(w, y)=m q$, we have $(3)$ holds.

Therefore, the desired result holds.

Combining Lemmas 6 and 7, we obtain the following theorem.

Theorem 8. $\Gamma_{q, s, k}$ is weakly distance-regular, if and only if $\mathrm{C} 1, \mathrm{C} 2$ or $\mathrm{C} 3$ holds.

Finally, we shall show that every weakly distance-regular digraph $\Gamma_{q, s, k}$ is a Cayley digraph.

Proposition 9. Let $d=\frac{p}{2 \operatorname{gcd}(q, p)}, l=\max \left\{w \mid 2^{w}\right.$ divides $\left.\operatorname{gcd}(q, p)\right\}, h=\frac{s}{2^{l}}, i=2\{d\}$ and $u$ be an integer such that $2^{i} q$ divides $(u p-\operatorname{gcd}(q, p))$, where $\{d\}$ denotes the fractional part of $d$ and $\operatorname{gcd}(q, p)$ denotes the greatest common divisor of $q$ and $p$.

(i) If $\mathrm{C} 1$ holds, then $\Gamma_{q, s, k}$ is isomorphic to $\operatorname{Cay}\left(\mathbb{Z}_{q} \times \mathbb{Z}_{2 m q},\{(1,0),(0,1),(1,2 m q-1)\}\right)$, $m \geqslant 1$ and $q \geqslant 3$.

(ii) If $\mathrm{C} 2$ holds, then $\Gamma_{q, s, k}$ is isomorphic to $\operatorname{Cay}\left(\mathbb{Z}_{(m q+2) q},\{1, m q+2, m q+1\}\right), m \geqslant 1$ and $q \geqslant 3$.

(iii) If C3 holds, then $\Gamma_{q, s, k}$ is isomorphic to $\mathrm{Cay}\left(\mathbb{Z}_{2^{i} q} \times \mathbb{Z}_{2^{-i}(2 m q+p)},\left\{\left(2^{i} u d, 1\right),\left(2^{i}\right.\right.\right.$ $\left.\left.\left.2^{i} u d, i h-1\right),\left(2^{i}, i h\right)\right\}\right)$, where $q \geqslant 3, m \geqslant 0,4 \leqslant p \leqslant 2 q-2$ and $p$ is even.

Proof. If C1 holds, then (i) is obvious. If $\mathrm{C} 2$ holds, then the mapping $\sigma$ from $\Gamma_{q, s, k}$ to the digraph in (ii) satisfying $\sigma(a, b)=\hat{a}(m q+2)+\hat{b}$ is an isomorphism.

Now suppose C3 holds. Let $\sigma$ be the mapping from $\Gamma_{q, s, k}$ to the digraph in (iii) such that $\sigma(a, b)=\left(2^{i} \hat{a}+2^{i} u d \hat{b}, i h \hat{a}+\hat{b}\right)$. Note that $\sigma$ is well defined.

We will show that $\sigma$ is injective. It is clear for $i=0$. If $i=1$, by $2 \mid p$, then $l \geqslant 1$. Assume that $\sigma\left(x_{1}, y_{1}\right)=\sigma\left(x_{2}, y_{2}\right)$ for $\left(x_{1}, y_{1}\right),\left(x_{2}, y_{2}\right) \in V \Gamma_{q, s, k}$. Let $x=2 u d\left(\widehat{y_{2}}-\widehat{y_{1}}\right)-2\left(\widehat{x_{1}}-\widehat{x_{2}}\right)$ and $y=\left(\widehat{y_{2}}-\widehat{y_{1}}\right)-h\left(\widehat{x_{1}}-\widehat{x_{2}}\right)$. Since $\sigma\left(x_{1}, y_{1}\right)=\sigma\left(x_{2}, y_{2}\right)$, we have $2 q \mid x$ and $(m q+p / 2) \mid y$, which imply $2^{l-1} h \mid y$ by $s=2 m q+p$. Hence, $h \mid\left(\widehat{y_{2}}-\widehat{y_{1}}\right)$.

We claim $2^{j} \mid\left(\widehat{y_{2}}-\widehat{y_{1}}\right)$ for $1 \leqslant j \leqslant l$. By $2 q \mid(u p-\operatorname{gcd}(q, p))$, we get $(2 q / \operatorname{gcd}(q, p)) \mid(2 u d-$ 1 ), which implies $2 u d$ is odd. Since $2 q \mid x$, one obtains $2 \mid\left(\widehat{y_{2}}-\widehat{y_{1}}\right)$. Suppose $2^{j} \mid\left(\widehat{y_{2}}-\widehat{y_{1}}\right)$ for some $j<l$. From $2^{j} \mid(m q+p / 2)$, we have $2^{j} \mid y$ and $\left.2^{j} \mid \widehat{x_{1}}-\widehat{x_{2}}\right)$, which imply $2^{j+1} \mid\left(\widehat{y_{2}}-\widehat{y_{1}}\right)$ by $2^{j+1} \mid x$. So our claim is valid.

By $\operatorname{gcd}\left(2^{l}, h\right)=1$, we obtain $(2 m q+p) \mid\left(\widehat{y_{2}}-\widehat{y_{1}}\right)$. Thus, $y_{1}=y_{2}$ and $x_{1}=x_{2}$. Therefore $\sigma$ is a bijection. One can verify that $\left(\left(x_{1}, y_{1}\right),\left(x_{2}, y_{2}\right)\right)$ is an arc if and only if $\left(\sigma\left(x_{1}, y_{1}\right), \sigma\left(x_{2}, y_{2}\right)\right)$ is an arc. Hence, $\sigma$ is an isomorphism. 


\section{Circuits}

In this section, we will discuss some properties for circuits of weakly distance-regular digraphs.

Let $\Gamma$ be a digraph. Let $R=\left\{\Gamma_{\widetilde{i}} \mid \widetilde{i} \in \widetilde{\partial}(\Gamma)\right\}$, where $\Gamma_{\widetilde{i}}=\{(x, y) \in V \Gamma \times V \Gamma \mid$ $\widetilde{\partial}(x, y)=\widetilde{i}\}$. If $\Gamma$ is weakly distance-regular, then $(V \Gamma, R)$ is an association scheme. For more information about association schemes, see [3, 11]. For two nonempty subsets $E$, $F \subseteq R$, define

$$
E F:=\left\{\Gamma_{\widetilde{h}} \mid \sum_{\Gamma_{\tilde{i}} \in E} \sum_{\Gamma_{\widetilde{j}} \in F} p_{\tilde{i}, \tilde{j}}^{\widetilde{h}} \neq 0\right\},
$$

and write $\Gamma_{\widetilde{i}} \Gamma_{\widetilde{j}}$ instead of $\left\{\Gamma_{\widetilde{i}}\right\}\left\{\Gamma_{\widetilde{j}}\right\}$. For each nonempty subset $F$ of $R$, define $\langle F\rangle$ to be the minimal equivalence relation containing $F$. Let

$$
V \Gamma / F:=\{F(x) \mid x \in V \Gamma\} \quad \text { and } \quad \Gamma_{\widetilde{i}}^{F}:=\left\{(F(x), F(y)) \mid y \in F \Gamma_{\widetilde{i}} F(x)\right\},
$$

where $F(x):=\left\{y \in V \Gamma \mid(x, y) \in \cup_{f \in F} f\right\}$. The digraph $\left(V \Gamma / F, \cup_{(1, s) \in \widetilde{\partial}(\Gamma)} \Gamma_{1, s}^{F}\right)$ is said to be the quotient digraph of $\Gamma$ over $F$, denoted by $\Gamma / F$. The size of $\Gamma_{\widetilde{i}}(x):=\{y \in V \Gamma \mid$ $\widetilde{\partial}(x, y)=\widetilde{i}\}$ depends only on $\widetilde{i}$, denoted by $k_{i}$. For any $(a, b) \in \widetilde{\partial}(\Gamma)$, we usually write $k_{a, b}$ (resp. $\left.\Gamma_{a, b}\right)$ instead of $k_{(a, b)}$ (resp. $\left.\Gamma_{(a, b)}\right)$.

Now we shall introduce some basic results which are used frequently in this paper.

Lemma 10. Let $\Gamma$ be a weakly distance-regular digraph. For each $\widetilde{i}:=(a, b) \in \widetilde{\partial}(\Gamma)$, define $\widetilde{i}^{*}=(b, a)$.

(i) $k_{\widetilde{h}} p_{i, \widetilde{j}}^{\widetilde{h}}=k_{\tilde{i}} p_{\widetilde{h}, \widetilde{j}^{*}}^{\widetilde{i}}=k_{\tilde{j}} p_{\tilde{i}^{*}, \widetilde{h}}^{\widetilde{j}}$.

(ii) $k_{\tilde{i}} k_{\tilde{j}}=\sum_{\widetilde{h} \in \widetilde{\partial}(\Gamma)} k_{\widetilde{h}} p_{\tilde{i}, \widetilde{j}}^{\widetilde{h}}$.

(iii) $\left|\Gamma_{\widetilde{i}} \Gamma_{\widetilde{j}}\right| \leqslant \operatorname{gcd}\left(k_{\tilde{i}}, k_{\tilde{j}}\right)$.

Proof. See Proposition 2.2 in [3, pp. 55-56] and Proposition 5.1 in [1].

In the remaining of this paper, we assume that $\Gamma$ is a weakly distance-regular digraph of valency 3 satisfying $k_{1, q-1}=1$ and $k_{1, g-1}=2$, where $q, g \geqslant 3$ and $q \neq g$. Let $A_{i, j}$ denote a binary matrix with rows and columns indexed by $V \Gamma$ such that $\left(A_{i, j}\right)_{x, y}=1$ if and only if $\widetilde{\partial}(x, y)=(i, j)$.

Lemma 11. The following hold:

$$
\begin{aligned}
& A_{1, q-1} A_{1, g-1}=A_{1, g-1} A_{1, q-1}, \\
& A_{1, g-1} A_{g-1,1}=A_{g-1,1} A_{1, g-1} .
\end{aligned}
$$

Proof. By Lemma 10 (iii), we may assume that

$$
A_{1, g-1} A_{1, q-1}=A_{i, j} \quad \text { and } \quad A_{1, q-1} A_{1, g-1}=A_{s, t}, \quad i, s \in\{1,2\} .
$$


We claim that $i=s=2$. Suppose $i=1$. Then $j=g-1$ because of $k_{1, q-1}=1$. By Lemma 10 (i), we get $p_{(g-1,1),(1, g-1)}^{(1, q-1)}=2 p_{(1, g-1),(1, q-1)}^{(1, g-1)}=2$. By Lemma 10 (iii), $A_{g-1,1} A_{1, g-1}=$ $2 I+2 A_{1, q-1}$, contrary to the fact that $A_{g-1,1} A_{1, g-1}$ is a symmetric matrix. Hence, $i=2$. Similarly, $s=2$ and our claim is valid.

Pick a path $\left(x_{0}, x_{1}, x_{2}\right)$ with $\widetilde{\partial}\left(x_{0}, x_{1}\right)=(1, g-1)$ and $\widetilde{\partial}\left(x_{1}, x_{2}\right)=(1, q-1)$. Then $\partial\left(x_{2}, x_{0}\right)=j$. We may choose a path $\left(x_{2}, x_{3}, \ldots, x_{j+1}, x_{0}\right)$. Since $\Gamma$ has just two types of arcs, there exists an $i \in\{1,2, \ldots, j+1\}$ such that $\widetilde{\partial}\left(x_{i}, x_{i+1}\right)=(1, q-1)$ and $\widetilde{\partial}\left(x_{i+1}, x_{i+2}\right)=(1, g-1)$, where $x_{j+2}=x_{0}$ and $x_{j+3}=x_{1}$. Since $\widetilde{\partial}\left(x_{i}, x_{i+2}\right)=(2, t)$, one has $t \leqslant j$. Similarly, $j \leqslant t$. Hence, $j=t$ and (4) holds.

In view of Lemma 10 (iii), we have

$$
\begin{aligned}
& A_{1, g-1} A_{g-1,1}=2 I+p_{(1, g-1),(g-1,1)}^{(s, s)} A_{s, s}, \quad s \geqslant 2, \\
& A_{g-1,1} A_{1, g-1}=2 I+p_{(g-1,1),(1, g-1)}^{(t, t)} A_{t, t}, \quad t \geqslant 2 .
\end{aligned}
$$

By Lemma 10 (ii), we have $k_{s, s} p_{(1, g-1),(g-1,1)}^{(s, s)}=k_{t, t} p_{(g-1,1),(1, g-1)}^{(t, t)}=2$, which implies that $p_{(1, g-1),(g-1,1)}^{(s, s)}, p_{(g-1,1),(1, g-1)}^{(t, t)} \in\{1,2\}$. Let $x_{0}$ and $x_{s}$ be two vertices satisfying $\widetilde{\partial}\left(x_{0}, x_{s}\right)=$ $(s, s)$. Suppose $p_{(1, g-1),(g-1,1)}^{(s, s)}=2$. Pick two distinct vertices $x, y \in P_{(1, g-1),(g-1,1)}\left(x_{0}, x_{s}\right)$. By $x_{0} \in P_{(g-1,1),(1, g-1)}(x, y)$ and $(7), \widetilde{\partial}(x, y)=(t, t)$. It follows that $p_{(g-1,1),(1, g-1)}^{(t, t)}=2$. Similarly, if $p_{(g-1,1),(1, g-1)}^{(t, t)}=2$, then $p_{(1, g-1),(g-1,1)}^{(s, s)}=2$ by $(6)$. Hence, $p_{(1, g-1),(g-1,1)}^{(s, s)}=$ $p_{(g-1,1),(1, g-1)}^{(t, t)}$. In order to show $(5)$, we shall prove $s=t$. Pick $x \in P_{(1, g-1),(g-1,1)}\left(x_{0}, x_{s}\right)$ and a path $P:=\left(x_{0}, x_{1}, \ldots, x_{s}\right)$.

Case 1. $P$ contains an arc of type $(1, g-1)$.

By (4), without loss of generality, we may assume that $\widetilde{\partial}\left(x_{0}, x_{1}\right)=(1, g-1)$. Pick $y \in \Gamma_{1, g-1}\left(x_{s}\right) \backslash\{x\}$. In view of $(7)$, if $x \neq x_{1}$, from $x_{0} \in P_{(g-1,1),(1, g-1)}\left(x_{1}, x\right)$, then $\partial\left(x_{1}, x\right)=t \leqslant s$; if $x=x_{1}$, from $x_{0} \in P_{(g-1,1),(1, g-1)}(x, y)$, then $\partial(x, y)=t \leqslant s$.

Case 2. $P$ only contains arcs of type $(1, q-1)$.

In this case, $A_{1, q-1}^{s} \neq I$. By (4), there exists a path $\left(x_{0}, y_{1}, y_{2}, \ldots, y_{s}, x\right)$ containing the unique arc $\left(x_{0}, y_{1}\right)$ of type $(1, g-1)$. If $x=y_{1}$, by Lemma 10 (iii), we have $A_{1, q-1}^{s}=I$, a contradiction. Therefore, $x \neq y_{1}$. By $x_{0} \in P_{(g-1,1),(1, g-1)}\left(y_{1}, x\right)$ and $(7)$, one has $\partial\left(y_{1}, x\right)=$ $t \leqslant s$.

Similarly, $t \geqslant s$, which implies $s=t$, as desired.

In the following, let $F=\left\langle\Gamma_{1, g-1}\right\rangle$ and fix $x \in V \Gamma$. Then $\Gamma / F$ is isomorphic to a circuit $C_{m}$ of length $m$. Let $\Delta$ be a digraph with the vertex set $F(x)$ such that $(y, z)$ is an arc of $\Delta$ if $(y, z)$ is an arc of type $(1, g-1)$ in $\Gamma$.

Lemma 12. Suppose that every circuit of length $g$ contains arcs of the same type in $\Gamma$. Then $\Delta_{t, g-t}\left(x_{0}\right)=\Gamma_{t, g-t}\left(x_{0}\right)$ for each $x_{0} \in F(x)$ and $t \in\{1,2, \ldots, g-1\}$.

Proof. Note that every arc of type $(1, g-1)$ is contained in a circuit of length $g$ with all arcs of type $(1, g-1)$. It follows that, for any such circuit $\left(x_{0}, x_{1}, \ldots, x_{g-1}\right)$, we have 
$\widetilde{\partial}_{\Gamma}\left(x_{0}, x_{i}\right)=(i, g-i)$, where $1 \leqslant i \leqslant g-1$. Then every arc of $\Delta$ is contained in a circuit of length $g$ in $\Delta$.

For any $x_{t} \in \Gamma_{t, g-t}\left(x_{0}\right)$, there exists a circuit $C_{g}:=\left(x_{0}, x_{1}, \ldots, x_{t}, \ldots, x_{g-1}\right)$ in $\Gamma$. Hence, $C_{g}$ only contains the arcs of same type. Suppose that each $\operatorname{arc}$ of $C_{g}$ is of type $(1, q-1)$. Then, $q<g$ and every circuit of length $q$ in $\Gamma$ only contains arcs of type $(1, q-1)$. It follows that $A_{1, q-1}^{q}=I$. Since $x_{0} \neq x_{l}$ for $1 \leqslant l \leqslant g-1, k_{1, q-1}=1$ implies that $g$ is the minimum positive integer such that $A_{1, q-1}^{g}=I$, a contradiction. Consequently, each arc of $C_{g}$ is of type $(1, g-1)$. Therefore, $\left(x_{0}, x_{t}\right) \in \Delta_{t, g-t}$; and so $\Gamma_{t, g-t}\left(x_{0}\right) \subseteq \Delta_{t, g-t}\left(x_{0}\right)$. Conversely, pick any $x_{t} \in \Delta_{t, g-t}\left(x_{0}\right)$. Then, in $\Gamma$, there exists a circuit $\left(x_{0}, x_{1}, \ldots, x_{t}, \ldots, x_{g-1}\right)$ each of whose arcs is of type $(1, g-1)$. Hence, $\left(x_{0}, x_{t}\right) \in \Gamma_{t, g-t}$; and so $\Delta_{t, g-t}\left(x_{0}\right) \subseteq \Gamma_{t, g-t}\left(x_{0}\right)$. Thus, the desired result holds.

Lemma 13. If $F(x)=V \Gamma$, then there exists a circuit of length $g$ containing different types of arcs.

Proof. Suppose for the contrary that every circuit of length $g$ contains the same type of arcs. By the Lemma 12, $\Gamma_{t, g-t}=\Delta_{t, g-t}$ for any $1 \leqslant t \leqslant g-1$. By (5), the proof of Proposition 4.3 in [8] implies that $\Delta$ is isomorphic to $\Gamma_{1}:=\operatorname{Cay}\left(\mathbb{Z}_{2 g},\{1, g+1\}\right)$ or $\Gamma_{2}:=\operatorname{Cay}\left(\mathbb{Z}_{g} \times \mathbb{Z}_{g},\{(0,1),(1,0)\}\right)$.

Case 1. $\Delta \simeq \Gamma_{1}$.

Choose $y \in \mathbb{Z}_{2 g} \backslash\{0,1, g+1\}$ and $t \in \mathbb{Z}_{2 g}$ such that $\widetilde{\partial}_{\Gamma}(0, y)=(1, q-1), \hat{t} \equiv \hat{y}(\bmod g)$ and $\hat{t} \in\{0,2,3, \ldots, g-1\}$. Since $(y+1, y+2, \ldots, y-t+g-1,0, y)$ is a path of length $g-\hat{t}, \partial_{\Gamma}(y+1, y)=g-1 \leqslant g-\hat{t}$. It follows that $t=0$, and so $\hat{y}=g$. Therefore, $\widetilde{\partial}_{\Gamma}(0, g)=(1, q-1)$. Similarly, $\widetilde{\partial}_{\Gamma}(g, 0)=(1, q-1)$. Hence, $q=2$, a contradiction.

Case 2. $\Delta \simeq \Gamma_{2}$.

Pick $(i, j) \in \Gamma_{1, q-1}(0,0)$. Since $\widetilde{\partial}_{\Delta}((0,0),(0, j))=(\hat{j}, g-\hat{j})$, by Lemma 12 , we have $\widetilde{\partial}_{\Gamma}((0,0),(0, j))=(\hat{j}, g-\hat{j})$. It follows that $i \neq 0$. By Lemma 10 (i), one gets $p_{(\hat{i}, g-\hat{i}),(\hat{j}, g-\hat{j})}^{(1, q-1)}=k_{\hat{i}, g-\hat{i}} p_{(1, q-1),(g-\hat{j}, \hat{j})}^{(\hat{i}, g-\hat{i})}$. Since $(i, j) \in P_{(1, q-1),(g-\hat{j}, \hat{j})}((0,0),(i, 0))$ in $\Gamma$, $p_{(1, q-1),(g-\hat{j}, \hat{j})}^{(\hat{i}, g-\hat{i})}=1$, which implies that $p_{(\hat{i}, g-\hat{i}),(\hat{j}, g-\hat{j})}^{(1, q-1)}=k_{\hat{i}, g-\hat{i}}$.

Let $\left((a, b),\left(a^{\prime}, b^{\prime}\right)\right)$ be an arc of type $(1, q-1)$. Then $P_{(\hat{i}, g-\hat{i}),(\hat{j}, g-\hat{j})}\left((a, b),\left(a^{\prime}, b^{\prime}\right)\right)=$ $\Gamma_{\hat{i}, g-\hat{i}}(a, b)$. Since $(a+i, b),(a, b+i) \in \Delta_{\hat{i}, g-\hat{i}}(a, b)$, by Lemma 12, $\left(a^{\prime}, b^{\prime}\right) \in \Gamma_{\hat{j}, g-\hat{j}}(a+i, b) \cap$ $\Gamma_{\hat{j}, g-\hat{j}}(a, b+i)$. By Lemma 12 again,

$$
\left(a^{\prime}, b^{\prime}\right) \in\{(a+i+j, b),(a+i, b+j)\} \cap\{(a+j, b+i),(a, b+i+j)\} .
$$

Since $i \neq 0$, we have $\left(a^{\prime}, b^{\prime}\right)=(a+i, b+j)=(a+j, b+i)$, which implies that $i=j$. Thus, $\Gamma \simeq \operatorname{Cay}\left(\mathbb{Z}_{g} \times \mathbb{Z}_{g},\{(1,0),(0,1),(i, i)\}\right)$. Since $g \neq q$, one gets $i \neq 1$. Let $g=n \hat{i}+r$ with $0 \leqslant r \leqslant \hat{i}-1$. If $r \neq 0$, then $\widetilde{\partial}_{\Gamma}((0,0),(1,1))=\widetilde{\partial}_{\Gamma}((0,0),(i, i+1))=(2, n+2 r-2)$; if $r=0$, then $\widetilde{\partial}_{\Gamma}((0,0),(1,1))=\widetilde{\partial}_{\Gamma}((0,0),(i, i+1))=(2, n+2 \hat{i}-3)$. But we have $(1,0) \in$ $P_{(1, g-1),(1, g-1)}((0,0),(1,1))$ and $P_{(1, g-1),(1, g-1)}((0,0),(i, i+1))=\emptyset$ in $\Gamma$, a contradiction.

Lemma 14. Every circuit of length $q$ in $\Gamma$ only contains the arcs of the same type. In particular,

$$
A_{1, q-1}^{2}=A_{2, q-2}
$$


Proof. If $F(x)=V \Gamma$, then $q<g$ by Lemma 13 and the desired result follows. Suppose $F(x) \neq V \Gamma$. Assume the contrary, namely, there exists a circuit $\left(x_{0}, x_{1}, \ldots, x_{q-1}\right)$ containing arcs of different types. Since $\Gamma / F \simeq C_{m}$ with $m \geqslant 2$, there exist at least two arcs of type $(1, q-1)$ in this circuit. By (4), we may assume that $\widetilde{\partial}\left(x_{0}, x_{1}\right)=\widetilde{\partial}\left(x_{1}, x_{2}\right)=(1, q-1)$ and $\widetilde{\partial}\left(x_{q-1}, x_{0}\right)=(1, g-1)$. By the claim in Lemma $11, \widetilde{\partial}\left(x_{q-1}, x_{1}\right)=(2, q-2)$. Since $k_{1, q-1}=1$, by Lemma 10 (ii), one has $k_{\widetilde{\partial}\left(x_{0}, x_{2}\right)}=1$. Therefore, $\widetilde{\partial}\left(x_{0}, x_{2}\right)=(2, q-2)$. But $P_{(1, q-1),(1, q-1)}\left(x_{0}, x_{2}\right)=\left\{x_{1}\right\}$ and $P_{(1, q-1),(1, q-1)}\left(x_{q-1}, x_{1}\right)=\emptyset$, a contradiction. Lemma 10 (iii) implies (8).

Lemma 15. For any circuit $\left(x_{0}, x_{1}, \ldots, x_{l-1}\right)$ with $\widetilde{\partial}\left(x_{l-1}, x_{0}\right)=(1, g-1)$, there exists $i \in\{0,1, \ldots, l-2\}$ such that $\widetilde{\partial}\left(x_{i}, x_{i+1}\right)=(1, g-1)$.

Proof. Suppose for the contradiction that $\widetilde{\partial}\left(x_{i}, x_{i+1}\right)=(1, q-1)$ for any $i=0,1, \ldots, l-2$. By Lemma 10 (iii), we have $A_{g-1,1}=A_{1, q-1}^{l-1}$. Then $A_{g-1,1}$ is a permutation matrix, a contradiction.

Lemma 16. $F(x) \neq V \Gamma$ if and only if every circuit of length $g$ in $\Gamma$ only contains the arcs of the same type.

Proof. Suppose $F(x) \neq V \Gamma$. Assume the contrary, namely, $\left(x_{0}, x_{1}, \ldots, x_{g-1}\right)$ is a circuit containing arcs of different types such that $\widetilde{\partial}\left(x_{0}, x_{1}\right)=(1, g-1)$. By (4) and Lemma 15, we may assume that $\widetilde{\partial}\left(x_{1}, x_{2}\right)=(1, q-1)$ and $\widetilde{\partial}\left(x_{g-1}, x_{0}\right)=(1, g-1)$. By the claim in Lemma $11, \widetilde{\partial}\left(x_{0}, x_{2}\right)=(2, g-2)$. If $\partial\left(x_{g-1}, x_{1}\right)=1$, from $F(x) \neq V \Gamma$, then $\widetilde{\partial}\left(x_{g-1}, x_{1}\right)=(1, g-1)$, which implies $\left(x_{1}, x_{2}, \ldots, x_{g-1}\right)$ is a circuit of length $g-1$ containing an arc of type $(1, g-1)$, a contradiction. Hence, $\widetilde{\partial}\left(x_{g-1}, x_{1}\right)=(2, g-2)$. The fact that $x_{2} \notin F\left(x_{0}\right)$ implies that $P_{(1, g-1),(1, g-1)}\left(x_{0}, x_{2}\right)=\emptyset$, contradicting to $x_{0} \in P_{(1, g-1),(1, g-1)}\left(x_{g-1}, x_{1}\right)$.

The converse is true by Lemma 13.

\section{The proof of Theorem 1}

In this section, we assume that $F=\left\langle\Gamma_{1, g-1}\right\rangle$ and $x$ is a fixed vertex of $\Gamma$.

Lemma 17. If $F(x) \neq V \Gamma$, then $\Gamma / F \simeq C_{2}$.

Proof. Suppose for the contradiction that $\Gamma / F \simeq C_{m}$ with $m \geqslant 3$. Choose a path $\left(x_{0}, x_{1}, x_{2}, x_{3}\right)$ such that $\widetilde{\partial}\left(x_{0}, x_{1}\right)=\widetilde{\partial}\left(x_{1}, x_{2}\right)=(1, q-1)$ and $\widetilde{\partial}\left(x_{2}, x_{3}\right)=(1, g-1)$. Since $\partial\left(F\left(x_{0}\right), F\left(x_{2}\right)\right)=2, k_{1, q-1}=1$ implies that $\widetilde{\partial}\left(x_{0}, x_{3}\right)=(3, l)$ for some $l$. Then there exists a shortest path $\left(x_{3}, x_{4}, y_{2}, \ldots, x_{l+2}, x_{0}\right)$. By Lemma 15 and (4), we may assume that $\widetilde{\partial}\left(x_{3}, x_{4}\right)=(1, g-1)$. Since $\partial\left(F\left(x_{1}\right), F\left(x_{4}\right)\right)=1$ and $k_{1, q-1}=1$, we have $\partial\left(x_{1}, x_{4}\right)=2$ or 3. If $\partial\left(x_{1}, x_{4}\right)=2$, by $F(x) \neq V \Gamma$, then $\widetilde{\partial}\left(x_{2}, x_{4}\right)=(1, g-1)$, which implies $g=2$ by $x_{2} \in P_{(g-1,1),(1, g-1)}\left(x_{3}, x_{4}\right)$ and $(7)$, a contradiction. Hence, $\widetilde{\partial}\left(x_{1}, x_{4}\right)=(3, t)$ for some $t \leqslant l$. From $m \geqslant 3$ and (4), there exists a path $\left(x_{4}, y_{1}, y_{2}, \ldots, y_{t-2}, x_{0}, x_{1}\right)$. Then $\left(x_{3}, x_{4}, y_{1}, y_{2}, \ldots, y_{t-2}, x_{0}\right)$ is a path of length $t$; and so $l \leqslant t$. Hence, $l=t$. By (8), $x_{2} \in P_{(2, q-2),(1, g-1)}\left(x_{0}, x_{3}\right)$. Then there exists $y \in P_{(2, q-2),(1, g-1)}\left(x_{1}, x_{4}\right)$. From $k_{1, q-1}=1$, $\widetilde{\partial}\left(x_{2}, y\right)=(1, q-1)$, which implies $\Gamma_{1, q-1} \in F$, a contradiction. 
Proposition 18. If $F(x) \neq V \Gamma$, then $\Gamma$ is isomorphic to one of the digraphs in Theorem 1 (i).

Proof. By Lemma 17, $V \Gamma$ has a partition $F(x) \dot{U} F\left(x^{\prime}\right)$. Let $\Delta$ and $\Delta^{\prime}$ be the subdigraphs of $\Gamma$ induced on $F(x)$ and $F\left(x^{\prime}\right)$, respectively. By (4) and $k_{1, q-1}=1, \sigma: F(x) \rightarrow F\left(x^{\prime}\right)$, $y \mapsto y^{\prime}$ is an isomorphism mapping from $\Delta$ to $\Delta^{\prime}$, where $y^{\prime} \in \Gamma_{1, q-1}(y)$. By Lemmas 12 and 16, $\Gamma_{r, g-r}(y)=\Delta_{r, g-r}(y)$ for each $y \in F(x)$ and $r \in\{1,2, \ldots, g-1\}$. By (5), the proof of Proposition 4.3 in [8] implies that $\Delta$ is isomorphic to $\Gamma_{1}:=\operatorname{Cay}\left(\mathbb{Z}_{g} \times \mathbb{Z}_{g},\{(1,0),(0,1)\}\right)$ or $\Gamma_{2}:=\operatorname{Cay}\left(\mathbb{Z}_{2 g},\{1, g+1\}\right)$. Suppose that $\tau_{i}$ is an isomorphism from $\Gamma_{i}$ to $\Delta$ if $\Gamma_{i}$ is isomorphic to $\Delta$.

We claim that $\Delta \simeq \Gamma_{2}$. Suppose for the contrary that $\Delta \simeq \Gamma_{1}$. Write $\tau_{1}(a, b)=(a, b, 0)$ and $\sigma(a, b, 0)=(a, b, 1)$ for each $(a, b) \in \mathbb{Z}_{g} \times \mathbb{Z}_{g}$. Let $((0,0,1),(c, d, 0))$ be an arc of type $(1, q-1)$. By $(8), \widetilde{\partial}_{\Gamma}((0,0,0),(c, d, 0))=(2, q-2)$. Lemma 12 implies that $c \neq 0$ and $d \neq 0$. By Lemma 12 again, we have $(c, d, 0) \in P_{(2, q-2),(g-\hat{d}, \hat{d})}((0,0,0),(c, 0,0))$ and $\widetilde{\partial}_{\Gamma}((0,0,0),(c, 0,0))=\widetilde{\partial}_{\Gamma}((0,0,0),(0, c, 0))$. By $k_{2, q-2}=1$, we have $(0, c, 0) \in$ $\Gamma_{g-\hat{d} \hat{d}}(c, d, 0)$. Then $(0, c, 0) \in\{(c, 0,0),(c-d, d, 0)\}$ by Lemma 12 . Hence, $c=d$.

Suppose $\hat{c}=g-1$. Since $((0,0,1),(-1,-1,0),(0,-1,0),(0,0,0))$ is a shortest path, $q=4$, contrary to Lemma 14. Suppose $\hat{c} \neq g-1$. Then $\widetilde{\partial}_{\Gamma}((0,0,0),(c, c+1,0))=(3, l)$ for some $l$. Pick a path $\left((c, c+1,0), x_{1}, x_{2}, \ldots, x_{l-1},(0,0,0)\right)$. By Lemma 15 and (4), we may assume that $\widetilde{\partial}_{\Gamma}\left((c, c+1,0), x_{1}\right)=(1, g-1)$. By $(7)$, we have $\widetilde{\partial}_{\Gamma}\left((0,0,1), x_{1}\right)=$ $(3, t)$ for some $t \leqslant l$. Since $F(x) \neq V \Gamma, k_{1, q-1}=1$ implies that there exists a path $\left(x_{1}, y_{1}, y_{2}, \ldots, y_{t-2},(0,0,0),(0,0,1)\right)$. Then $\left((c, c+1,0), x_{1}, y_{1}, y_{2}, \ldots, y_{t-2},(0,0,0)\right)$ is a path of length $t$; and so $l \leqslant t$. Hence $l=t$. By (8) and $x_{1} \in V \Delta$, one has $(c, c, 0) \in$ $P_{(2, q-2),(1, g-1)}((0,0,0),(c, c+1,0))$ and $P_{(2, q-2),(1, g-1)}\left((0,0,1), x_{1}\right)=\emptyset$ in $\Gamma$, a contradiction. Therefore, our claim is valid.

Write $\tau_{2}(a)=(a, 0)$ and $\sigma(a, 0)=(a, 1)$ for each $a \in \mathbb{Z}_{2 g}$. Let $\left((a, 1),\left(a+k_{a}, 0\right)\right)$ be an arc of type $(1, q-1)$. Then $k_{a} \neq 0$. By $(8), \widetilde{\partial}_{\Gamma}\left((a, 0),\left(a+k_{a}, 0\right)\right)=(2, q-2)$. By Lemma 12 , $\widetilde{\partial}_{\Delta}\left((a, 0),\left(a+k_{a}, 0\right)\right) \neq(t, g-t)$ for any $t \in\{1,2, \ldots, g-1\}$. Since $\bigcup_{1 \leqslant t \leqslant g-1} \Delta_{t, g-t}(a, 0)=$ $V \Delta \backslash\{(a, 0),(a+g, 0)\}$, one has $\widehat{k_{a}}=g$. Then, $\Gamma \simeq \operatorname{Cay}\left(\mathbb{Z}_{4} \times \mathbb{Z}_{g},\{(0,1),(1,0),(2,1)\}\right)$ and the result holds by Proposition 2.

Lemma 19. If $F(x)=V \Gamma$, then $p_{(1, g-1),(1, g-1)}^{(1, q-1)}=2$.

Proof. By Lemma 13, there exists a circuit of length $g$ with different types of arcs. Let $C:=\left(x_{0}, x_{1}, \ldots, x_{g-1}\right)$ be such a circuit with the minimum number of arcs of type $(1, g-1)$. Suppose $C$ contains $t$ arcs of types $(1, g-1)$. Lemma 15 implies that $t \geqslant 2$. By (4), we may assume that $\widetilde{\partial}\left(x_{i}, x_{i+1}\right)=(1, g-1)$ for $0 \leqslant i \leqslant t$. We claim that $\widetilde{\partial}\left(x_{0}, x_{2}\right)=(1, q-1)$. Suppose not. By the claim in Lemma 11 and (7), we have $\widetilde{\partial}\left(x_{g-1}, x_{1}\right)=\widetilde{\partial}\left(x_{0}, x_{2}\right)=$ $(2, g-2)$. Since $x_{0} \in P_{(1, q-1),(1, g-1)}\left(x_{g-1}, x_{1}\right)$, there exists $x_{1}^{\prime} \in P_{(1, q-1),(1, g-1)}\left(x_{0}, x_{2}\right)$. The circuit $C^{\prime}:=\left(x_{0}, x_{1}^{\prime}, x_{2}, \ldots, x_{g-1}\right)$ contains just $t-1$ arcs of type $(1, g-1)$, a contradiction. Thus, our claim is valid. It follows that $p_{(1, q-1),(g-1,1)}^{(1, g-1)}=1$. By Lemma $10(\mathrm{i})$, the desired result holds. 
Let $H=\left\langle\Gamma_{1, q-1}\right\rangle$ and $H\left(x_{0,0}\right), H\left(x_{0,1}\right), \ldots, H\left(x_{0, s-1}\right)$ be all pairwise distinct vertices of $\Gamma / H$. Since $q<g$, the subdigraph induced on each $H\left(x_{0, j}\right)$ is a circuit of length $q$ with arcs of type $(1, q-1)$, say $\left(x_{0, j}, x_{1, j}, \ldots, x_{q-1, j}\right)$. It follows that $s \geqslant 2$.

Proposition 20. If $F(x)=V \Gamma$, then $\Gamma$ is isomorphic to one of the digraphs in Theorem 1 (ii).

Proof. Suppose $\partial\left(H\left(x_{0,0}\right), H\left(x_{0,1}\right)\right)=1$. By (4), we may assume that $\widetilde{\partial}\left(x_{0,0}, x_{0,1}\right)=(1, g-$ 1). By Lemma 19 , one has $\widetilde{\partial}\left(x_{0,1}, x_{1,0}\right)=(1, g-1)$, which implies $\partial\left(H\left(x_{0,1}\right), H\left(x_{0,0}\right)\right)=1$. Since $F(x)=V \Gamma, \Gamma / H$ is a connected undirected graph. By $k_{1, g-1}=2, \Gamma / H$ is an undirected circuit of length $s$. Suppose $s=2$. Pick $y \in \Gamma_{1, g-1}\left(x_{0,1}\right) \backslash\left\{x_{1,0}\right\}$. Then $y=x_{i, 0}$ for some $i \geqslant 2$, and $\left(x_{0,1}, y, x_{i+1,0}, \ldots, x_{q-1,0}, x_{0,0}\right)$ is a path of length $q-i+1$ from $x_{0,1}$ to $x_{0,0}$, contrary to the fact $\partial\left(x_{0,1}, x_{0,0}\right)=g-1$. Hence, $s \geqslant 3$.

Let $\left(H\left(x_{0,0}\right), H\left(x_{0,1}\right), \ldots, H\left(x_{0, s-1}\right)\right)$ be an undirected circuit. By $(4)$, we may assume that $\left(x_{0,0}, x_{0,1}, \ldots, x_{0, s-1}\right)$ is a path with arcs of type $(1, g-1)$. By Lemma 19, $\left(x_{0, j}, x_{0, j+1}, x_{1, j}, x_{1, j+1}, x_{2, j}, \ldots, x_{q-1, j}, x_{q-1, j+1}\right)$ is a circuit with arcs of type $(1, g-1)$ for $j=0,1, \ldots, s-2$. Hence, there exists $k \in\{1,2, \ldots, q\}$ such that $\widetilde{\partial}\left(x_{0, s-1}, x_{q-k+1,0}\right)=$ $(1, g-1)$, where the first subscription of $x$ are taken modulo $q$. By Lemma 19 again, we obtain $\widetilde{\partial}\left(x_{i, s-1}, x_{i-k+1,0}\right)=\widetilde{\partial}\left(x_{i-k+1,0}, x_{i+1, s-1}\right)=(1, g-1)$ for each $i$. Since

$$
\left(x_{0,0}, x_{0,1}, \ldots, x_{0, s-1}, x_{q-k+1,0}, x_{q-k+2,0}, \ldots, x_{q-1,0}\right)
$$

is a circuit of length $s+k-1$ with different types of arcs, by Lemma 14, we get $s+k-1>q$. From Theorem 8, the desired result follows.

Combining Propositions 18 and 20, we complete the proof of Theorem 1.

In the forthcoming paper [10], we shall classify cubic commutative weakly distanceregular digraphs with one type of arcs.

\section{Acknowledgements}

We are grateful to the referee for useful comments and suggestions. This research is supported by NSFC (11271047, 11301270, 11371204, 11501036), the Fundamental Research Funds for the Central University of China, Youth Scholar Program of Beijing Normal University and China Postdoctoral Science Foundation (2015M570958).

\section{References}

[1] Z. Arad, E. Fisman and M. Muzychuk. Generalized table algebras. Israel J. Math., 114:29-60, 1999.

[2] E. Bannai, P.J. Cameron and J. Kahn. Nonexistence of certain distance-transitive digraphs. J. Combin. Theory Ser. B, 31:105-110, 1981.

[3] E. Bannai and T. Ito. Algebraic Combinatorics I: Association Schemes. Benjamin/Cummings, California, 1984. 
[4] R.M. Damerell. Distance-transitive and distance regular digraphs. J. Combin. Theory Ser. B, 31:46-53, 1981.

[5] C.W. Lam. Distance-transitive digraphs. Discrete Math., 29:265-274, 1980.

[6] D.A. Leonard and K. Nomura. The girth of a directed distance-regular digraph. J. Combin. Theory Ser. B, 58:34-39, 1993.

[7] H. Suzuki. Thin weakly distance-regular digraphs. J. Combin. Theory Ser. B, 92:6983, 2004.

[8] K. Wang and H. Suzuki. Weakly distance-regular digraphs. Discere Math., 264:225236, 2003.

[9] K. Wang. Weakly distance-regular digraphs of girth 2, European J. Combin., 25:363$375,2004$.

[10] Y. Yang, B. Lv and K. Wang. Weakly distance-regular digraphs of valency three, II, in preparation.

[11] P.H. Zieschang. An Algebraic Approach to Assoication Schemes. In Lecture Notes in Mathematics, Vol.1628, Springer, Berlin-Heidelberg, 1996. 